\title{
Singularity functions for fractional processes: application to the fractional Brownian sheet
}

\author{
Serge Cohen ${ }^{\mathrm{a}, *}$, Xavier Guyon ${ }^{\mathrm{b}}$, Olivier Perrin ${ }^{\mathrm{c}}$, Monique Pontier $^{\mathrm{a}}$ \\ ${ }^{a}$ LSP, Université Paul Sabatier, 118, route de Narbonne, 31062 Toulouse cedex 04, France \\ b SAMOS, Université Paris I, 90, rue de Tolbiac, 75634 Paris cedex 13, France \\ ${ }^{\mathrm{c}}$ GREMAQ, Université Toulouse 1 and LERNA, INRA, 21, allée de Brienne, 31000 Toulouse, France
}

Received 22 March 2004; received in revised form 24 November 2004; accepted 15 March 2005

Available online 22 June 2005

\begin{abstract}
In this paper almost sure convergence and asymptotic normality of generalized quadratic variation are studied. The main result in this paper extend classical results from Baxter and Gladyshev so that they can be applied to fractional Gaussian processes. An application to the estimation of the true axes of a fractional Brownian sheet is also obtained.

(c) 2005 Elsevier SAS. All rights reserved.

\section{Résumé}

On étudie dans ce papier les propriétés de convergence et de normalité asymptotique des variations quadratiques généralisées d'un champ brownien fractionnaire. Le résultat principal est une extension des résultats classiques de Baxter et Gladyshev au cas de processus gaussiens fractionnaires. Nous appliquons ce résultat à l'estimation de la direction privilégiée de tels processus. () 2005 Elsevier SAS. All rights reserved.
\end{abstract}

MSC: primary 60G15, 60G60; secondary $62 \mathrm{H} 11,62 \mathrm{H} 12$

Keywords: Fractional Brownian sheet; Estimation; Anisotropy; Generalized quadratic variations

\section{Introduction}

Since [2] it is classical to show that quadratic variations of a Gaussian process converge to an integral of the singularity function if it exists. This result is extended in [8] to some fractional processes including the well known fractional Brownian motion. In [8] the mesh of the increments that define the quadratic variation is $1 / 2^{n}$ and an al-

\footnotetext{
* Corresponding author.

E-mail addresses: serge.cohen@math.ups-tlse.fr (S. Cohen), guyon@univ-paris1.fr (X. Guyon), perrin@ cict.fr (O. Perrin), monique.pontier@math.ups-tlse.fr (M. Pontier).
} 
most sure convergence result is obtained when $n \rightarrow \infty$. Actually it is known (see [12]) that the almost convergence is true when the mesh is $\mathrm{o}(1 / \log (n))$. Nevertheless quadratic variations are not suitable when one is interested in a central limit theorem for fractional Brownian motion. Actually it is shown in [9] that for $H>3 / 4$ the central limit theorem is false for fractional Brownian motion. To overcome this problem the usual increments in the quadratic variations are replaced by second order increments to yield the generalized quadratic variations. In this case generalized quadratic variations have been used in [10] for Gaussian processes with stationary increments and in [3] for filtered white noise that are fractional processes with non-stationary increments.

In the first part of the paper the almost sure convergence of the generalized quadratic variations is obtained under general assumption on the correlation of the Gaussian process when the mesh is $1 / n$. When the limit is not vanishing it leads to a generalization of the singularity function in the spirit of Gladyshev. Then the asymptotic normality of the generalized quadratic variations is established in this general setting. In the second part an example of application of this theorem to spatial estimation for a Gaussian field is given. Actually we consider a fractional Brownian sheet which is a centered Gaussian field $\left(W_{H_{1}, H_{2}}(x, y)\right)_{(x, y) \in \mathbb{R}^{2}}$ such that the correlation function is:

$$
E\left[W\left(x_{1}, y_{1}\right) \cdot W\left(x_{2}, y_{2}\right)\right]=\frac{1}{4}\left(\left|x_{1}\right|^{2 H_{1}}+\left|x_{2}\right|^{2 H_{1}}-\left|x_{1}-x_{2}\right|^{2 H_{1}}\right)\left(\left|y_{1}\right|^{2 H_{2}}+\left|y_{2}\right|^{2 H_{2}}-\left|y_{1}-y_{2}\right|^{2 H_{2}}\right)
$$

where $0<H_{1}, H_{2}<1$.

With no loss of generality one can assume that the $O x$ axis is the one corresponding to the minimum of $H_{1}, H_{2}$; hence, we denote by $H_{1}$ this minimum from now on. This field has been introduced in [11] and studied in [7,1]. The estimation of the parameter $H_{1}, H_{2}$ is done in [13] under the assumption that the axes of the fractional Brownian sheet are known. We refer to [13] for a discussion of the application of this estimation to the detection of osteoporosis with medical X-rays.

In our paper the parameters $H_{1}, H_{2}$ are identified with the help of generalized quadratic variations of the fractional Brownian sheet restricted to some segments and we do not assume that the axes of the fractional Brownian sheet are known as in [13]. Please note that the use of generalized quadratic variations is quite important because one constructs a confidence interval with the help of the central limit theorem. Moreover we address the problem of estimating the rotation between the axes of the fractional Brownian sheet and the axes of the observations. Please note that a similar problem is solved in [5] for standard Brownian sheet i.e. $H_{1}=H_{2}=1 / 2$ (see [14] for a general reference).

Strongly consistent estimators of $H_{1}, H_{2}$ and $\theta$ the angle between the axes of observations and the true axes of the fractional Brownian sheet are given. Moreover a confidence interval is obtained for $H_{1}$.

In the second section almost sure convergence of the generalized quadratic variations is established, a Central Limit Theorem is also obtained. Section 3 is devoted to the application to the fractional Brownian sheet.

\section{Singularity functions for fractional processes}

In the following theorem the almost sure convergence of the generalized quadratic variations is proved. Let us stress the fact that this theorem is classical for quadratic variations (cf. [8]) in [8] a bound for the second derivative of the correlation function $r(s, t)$ is needed when $s \neq t$. In the following theorem this bound is replaced by (3) which is a bound on the fourth derivative of the covariance function $r(s, t)$. Moreover the singularity function (see Remark 2 for a precise definition) is now obtained in (6) as a limit of a fourth order difference operator applied to $r$. Those are the key points for describing the limit behavior of the generalized quadratic variations.

Theorem 1. Let $\left(\xi_{t}, t \in[0,1]\right)$ be a real process with Gaussian increments such that

(1) $m_{t}=\mathbb{E}\left(\xi_{t}\right)$ exists and has a bounded derivative on the segment $[0,1]$. 
(2) There exists a correlation function

$$
r(s, t)=\mathbb{E}((\xi(s)-\mathbb{E} \xi(s))(\xi(t)-\mathbb{E} \xi(t)))
$$

having the following properties:

(a) $r$ is continuous on $[0,1]^{2}$;

(b) There exist two function $c_{1}(s), c_{2}(t)$ such that $\partial^{4}\left(r-c_{1}-c_{2}\right) / \partial t^{2} \partial s^{2}$ exists and is a continuous function on $[0,1]^{2} \backslash$ Diag, where Diag $=\{(u, v)$ : such that $u=v\}$ and there exists a constant $C_{0}$ and a real number $\gamma \in(0,2)$ such that

$$
\left|\frac{\partial^{4}\left(r-c_{1}-c_{2}\right)(s, t)}{\partial t^{2} \partial s^{2}}\right| \leqslant \frac{C_{0}}{|s-t|^{\gamma+2}} .
$$

(c) Let us define two order increments:

$$
\begin{aligned}
& \delta_{1}^{h} f(s, t)=f(s+h, t)+f(s-h, t)-2 f(s, t), \\
& \delta_{2}^{h} f(s, t)=f(s, t+h)+f(s, t-h)-2 f(s, t)
\end{aligned}
$$

and let us suppose that there exists a bounded function $g$ defined on $(0,1)$ such that:

$$
\lim _{h \rightarrow 0^{+}} \sup _{t \in[h, 1-h]}\left|\frac{\left(\delta_{1}^{h} \circ \delta_{2}^{h} r\right)(t, t)}{h^{2-\gamma}}-g(t)\right|=0 .
$$

Then

$$
\lim _{N \rightarrow \infty} N^{1-\gamma} \sum_{k=1}^{N-1}\left[\xi_{(k+1) / N}+\xi_{(k-1) / N}-2 \xi_{k / N}\right]^{2}=\int_{0}^{1} g(t) \mathrm{d} t .
$$

Remark 1. Please remark that $g$ is continuous on $(0,1)$ because of $(6)$.

Remark 2. If assumption (2b) and assumption (2c) are satisfied for $\gamma_{0} \in(0,2)$, they are also satisfied for $\gamma>\gamma_{0}$ but the corresponding function $g_{\gamma}$ is vanishing. When $\gamma_{0}$ is the infimum of the real number such that assumptions (2b) and (2c) are satisfied $g_{\gamma_{0}}$ can be viewed as a generalization of the singularity function introduced in [2].

Remark 3. In assumption (2b) the functions $c_{1}(s), c_{2}(t)$ are introduced so that Theorem 1 can be applied to the fractional Brownian motion with covariance

$$
r(s, t)=\frac{1}{2}\left\{|s|^{2 H}+|t|^{2 H}-|t-s|^{2 H}\right\}
$$

where $0<H<1$. Actually the partial derivatives of the covariance $r$ do not exist when $s=0$ but assumption (2b) still holds. Assumption (2a) is clearly fulfilled for the fractional Brownian motion. Since

$$
\begin{aligned}
\delta_{1}^{h} \circ \delta_{2}^{h} r(t, t)= & 4 r(t, t)+2 r(t-h, t+h)-4 r(t+h, t)-4 r(t-h, t) \\
& +r(t+h, t+h)+r(t-h, t-h),
\end{aligned}
$$

we get $\delta_{1}^{h} \circ \delta_{2}^{h} r(t, t)=\left(4-2^{2 H}\right) h^{2 H}$. If one makes the choice $\gamma=2-2 H$ then $\forall t \in[h, 1-h] g(t)=\left(4-2^{2 H}\right)$, assumption $(2 \mathrm{c})$ is fulfilled.

Proof of Theorem 1. One can suppose that $m(t)=0$ for $t \in[0,1]$ by considering the process $\tilde{\xi}(t)=\xi(t)-\mathbb{E} \xi(t)$. Because of assumption 1 , as $N \rightarrow \infty$

$$
N^{1-\gamma} \sum_{k=1}^{N-1}\left[m_{(k+1) / N}+m_{(k-1) / N}-2 m_{k / N}\right]^{2}=\mathrm{O}\left(N^{-\gamma}\right),
$$


the existence of the limit (7) yields the result for a noncentered $\xi(t)$. We suppose that $m(t)=0$ for $t \in[0,1]$ until the end of the proof.

Let us define $\Delta \xi_{k}=\xi_{(k+1) / N}+\xi_{(k-1) / N}-2 \xi_{k / N}$, and

$$
\eta_{N}=N^{1-\gamma} \sum_{k=1}^{N-1}\left(\Delta \xi_{k}\right)^{2} .
$$

The following notations are introduced:

$$
d_{j, k}=N^{1-\gamma} \mathbb{E}\left[\Delta \xi_{j} \Delta \xi_{k}\right]
$$

and

$$
d_{N}=2 \sum_{k=1}^{N-1} d_{k, k}^{2}+4 \sum_{1 \leqslant k<j \leqslant N-1} d_{j, k}^{2} .
$$

Let us first prove $d_{N}=\operatorname{var}\left(\eta_{N}\right)$. Actually

$$
\eta_{N}^{2}=N^{2-2 \gamma}\left[\sum_{k=1}^{N-1}\left(\Delta \xi_{k}\right)^{4}+2 \sum_{1 \leqslant k<j \leqslant N-1}\left(\Delta \xi_{k}\right)^{2}\left(\Delta \xi_{j}\right)^{2}\right] .
$$

Since $\left(\Delta \xi_{k}, k=1, \ldots, N-1\right)$ is a Gaussian vector

$$
\mathbb{E}\left[\left(\Delta \xi_{k}\right)^{4}\right]=3\left(\mathbb{E}\left[\left(\Delta \xi_{k}\right)^{2}\right]\right)^{2}
$$

and

$$
\mathbb{E}\left[\left(\Delta \xi_{k}\right)^{2}\left(\Delta \xi_{j}\right)^{2}\right]=\mathbb{E}\left[\left(\Delta \xi_{k}\right)^{2}\right] \mathbb{E}\left[\left(\Delta \xi_{j}\right)^{2}\right]+2\left(\mathbb{E}\left[\Delta \xi_{k} \Delta \xi_{j}\right]\right)^{2} .
$$

Then

$$
\mathbb{E}\left[\eta_{N}^{2}\right]=3 \sum_{k=1}^{N-1} d_{k, k}^{2}+2 \sum_{1 \leqslant k<j \leqslant N-1}\left[d_{k, k} d_{j, j}+2 d_{j, k}^{2}\right]
$$

that yields

$$
\operatorname{var}\left(\eta_{N}\right)=2 \sum_{k=1}^{N-1} d_{k, k}^{2}+4 \sum_{1 \leqslant k<j \leqslant N-1} d_{j, k}^{2}=d_{N} .
$$

Moreover we will show that there exists a generic constant $C$ such that

$$
\mathbb{E}\left[\left(\eta_{N}-\mathbb{E}\left(\eta_{N}\right)\right)^{4}\right] \leqslant C d_{N}^{2} .
$$

Let us remark that $\eta_{N}$ is the square of the Euclidean norm of the Gaussian vector $N^{(1-\gamma) / 2}\left(\Delta \xi_{k}, k=1, \ldots\right.$, $N-1)$. The classical Cochran theorem yields $k_{N} \leqslant N-1$ non-negative real numbers $\left(\lambda_{1, N}, \ldots, \lambda_{k_{N}, N}\right)$ and a $k_{N}$-dimensional Gaussian vector $\zeta$ such that its components are independent reduced Gaussian variables and

$$
\eta_{N}=\sum_{j=1}^{k_{N}} \lambda_{j, N} \zeta_{j, N}^{2}
$$

The components of $\zeta$ are obtained by a simple orthogonalization procedure. Then 


$$
\begin{aligned}
\mathbb{E}\left[\left(\eta_{N}-\mathbb{E}\left(\eta_{N}\right)\right)^{4}\right] & =E\left[\left(\sum_{j=1}^{k_{N}} \lambda_{j, N}\left(\zeta_{j, N}^{2}-1\right)\right)^{4}\right] \\
& =\mathbb{E}\left[\left(\zeta_{1, N}^{2}-1\right)^{4}\right] \sum_{j=1}^{k_{N}} \lambda_{j, N}^{4}+6\left(\mathbb{E}\left[\left(\zeta_{1, N}^{2}-1\right)^{2}\right]\right)^{2} \sum_{1 \geqslant i<j \geqslant k_{N}} \lambda_{i, N}^{2} \lambda_{j, N}^{2} \\
& \leqslant C\left[\sum_{j=1}^{k_{N}} \lambda_{j, N}^{2}\right]^{2} .
\end{aligned}
$$

Moreover $d_{N}=\operatorname{var}\left(\eta_{N}\right)=\mathbb{E}\left[\left(\zeta_{1, N}^{2}-1\right)^{2}\right] \sum_{j=1}^{k_{N}} \lambda_{j, N}^{2}$ yields the inequality (13).

Let us remark that

$$
d_{j, k}=N^{1-\gamma}\left(\delta_{1}^{1 / N} \circ \delta_{2}^{1 / N} r\right)\left(\frac{j}{N}, \frac{k}{N}\right)
$$

and that

$$
\mathbb{E}\left(\eta_{N}\right)=\sum_{k=1}^{N-1} d_{k, k} .
$$

Moreover the limit (6) yields

$$
\sup _{1 \leqslant k \leqslant N-1}\left|N d_{k, k}-g\left(\frac{k}{N}\right)\right| \rightarrow 0
$$

as $N \rightarrow \infty$. Hence, for $M>\sup _{t \in(0,1)}|g(t)|$

$$
\left|d_{k, k}\right|<\frac{M}{N}
$$

for $N$ large enough.

Please remark that $g$ is continuous (Remark 1) so that

$$
\lim _{N \rightarrow \infty} \frac{1}{N} \sum_{k=1}^{N} g\left(\frac{k}{N}\right)=\int_{0}^{1} g(t) \mathrm{d} t .
$$

So we get

$$
\lim _{N \rightarrow \infty} \mathbb{E}\left(\eta_{N}\right)=\int_{0}^{1} g(t) \mathrm{d} t .
$$

The next step is to apply Borel-Cantelli lemma. To prove the almost sure convergence of $\eta_{N}$, an estimate of the asymptotic of $d_{N}$ when $N \rightarrow \infty$ is needed. Let us split the study of the asymptotic of (12) in three parts.

- We know that $d_{k, k}<M / N$ (cf. (17)), then

$$
\sum_{k=1}^{N-1} d_{k, k}^{2}<\frac{M^{2}}{N}
$$

for $M>\sup _{t \in(0,1)}|g(t)|$ and $N$ large enough. 
- For $j=k+1, k+2$, as $\left|d_{j, k}\right| \leqslant \sqrt{d_{k, k} d_{j, j}}$, we have

$$
\left|d_{j, k}\right| \leqslant \frac{M}{N}
$$

for $M>\sup _{t \in(0,1)}|g(t)|$ and $N$ large enough.

- A sharper estimate is needed for $|j-k|>2$.

Lemma 1. If $[s-h, s+h] \times[t-h, t+h] \cap$ Diag $=\emptyset$, where Diag $=\{(u, v)$ : such that $u=v\}$, and $[s-h$, $s+h] \times[t-h, t+h] \subset[0,1]^{2}$, then

$$
\left|\left(\delta_{1}^{h} \circ \delta_{2}^{h} r\right)(s, t)\right| \leqslant \frac{C_{0} h^{4}}{|s-t-2 h|^{\gamma+2}}
$$

for the constant $C_{0}$ of (3).

Proof. One can rewrite

$$
\delta_{1}^{h} \circ \delta_{2}^{h}\left(r-c_{1}-c_{2}\right)(s, t)=\int_{s}^{s+h} \mathrm{~d} u \int_{u-h}^{u} \mathrm{~d} x \int_{t}^{t+h} \mathrm{~d} v \int_{v-h}^{v} \mathrm{~d} y \frac{\partial^{4}\left(r-c_{1}-c_{2}\right)}{\partial s^{2} \partial t^{2}}(x, y) .
$$

Because of (3)

$$
\left|\frac{\partial^{4}\left(r-c_{1}-c_{2}\right)}{\partial s^{2} \partial t^{2}}(x, y)\right|<\frac{C_{0}}{|x-y|^{\gamma+2}}
$$

on the set $\{s-h \leqslant u-h \leqslant x \leqslant u \leqslant s+h ; t-h \leqslant v-h \leqslant y \leqslant v \leqslant t+h\}$ where $|x-y|>|s-t-2 h|$. Last $\delta_{1}^{h} \circ \delta_{2}^{h}\left(r-c_{1}-c_{2}\right)(s, t)=\left(\delta_{1}^{h} \circ \delta_{2}^{h} r\right)(s, t)$.

One can deduce from the preceding lemma that for $|j-k|>2$

$$
\left|d_{j, k}\right| \leqslant \frac{C_{0} N^{-1}}{|j-k-2|^{\gamma+2}} .
$$

Hence for $N \geqslant 5$

$$
\begin{aligned}
\sum_{1 \leqslant k \leqslant j-3 \leqslant N-4} d_{j, k}^{2} & \leqslant C_{0}^{2} N^{-2} \sum_{1 \leqslant k \leqslant j-3 \leqslant N-4}|j-k-2|^{-2 \gamma-4} \\
& =C_{0}^{2} N^{-2} \sum_{t=1}^{N-4} \frac{N-3-t}{t^{2 \gamma+4}} \\
& \leqslant C_{0}^{2} N^{-1}\left(\int_{1}^{N} \frac{\mathrm{d} t}{t^{2 \gamma+4}}+1\right) \\
& \leqslant \frac{4 C_{0}^{2}}{3 N}
\end{aligned}
$$

Since the number of terms that satisfy (20) is bounded by $2 N$, because of (12), of (19) and of (27) we get

$$
N d_{N}<10 M^{2}+\frac{16}{3} C_{0}^{2}
$$

for $M>\sup _{t \in(0,1)}|g(t)|$ and $N$ large enough. 
Because of (13), for $\delta>0$

$$
\mathbb{P}\left\{\left|\eta_{N}-\mathbb{E}\left(\eta_{N}\right)\right|>N^{(\delta-1) / 4}\right\} \leqslant N^{1-\delta} C d_{N}^{2}
$$

by Borel-Cantelli lemma for $\delta \in(0,1)$, one can deduce

$$
\lim _{N \rightarrow \infty}\left(\eta_{N}-\mathbb{E}\left(\eta_{N}\right)\right)=0 \quad \text { a.s. }
$$

and the convergence (7) comes from (18).

Let us now state some central limit theorems.

Theorem 2. Under the assumptions of Theorem 1 , if $m(t)=0 \forall t \in[0,1]$, and if $g(t)$ is a non-vanishing function

$$
\frac{N^{1-\gamma}\left[\sum_{k=1}^{N-1} \Delta \xi_{k}^{2}-\mathbb{E} \sum_{k=1}^{N-1} \Delta \xi_{k}^{2}\right]}{\sqrt{d}_{N}}
$$

converges to a standard centered Gaussian variable, as $N \rightarrow+\infty$.

Proof. Let us recall

$$
\eta_{N}=\sum_{j=1}^{k_{N}} \lambda_{j, N} \zeta_{j, N}^{2}
$$

the central limit theorem with Lindeberg condition is applied to

$$
S_{N}=\sum_{j=1}^{k_{N}} \lambda_{j, N}\left(\zeta_{j, N}^{2}-1\right)
$$

Hence we have to check that $\max _{1 \leqslant j \leqslant k_{N}} \lambda_{j, N}=\mathrm{o}\left(\sqrt{\operatorname{var}\left(S_{N}\right)}\right)$. This last condition is a consequence of an elementary result in linear algebra:

$$
\max _{1 \leqslant j \leqslant k_{N}} \lambda_{j, N} \leqslant \max _{1 \leqslant j \leqslant N-1} \sum_{i=1}^{N-1} N^{1-\gamma}\left|\mathbb{E}\left(\Delta \xi_{i} \Delta \xi_{j}\right)\right| .
$$

We already know estimates on the upper bound:

$$
\begin{aligned}
N^{1-\gamma} \max _{1 \leqslant j \leqslant N-1} \sum_{i=1}^{N-1}\left|\mathbb{E}\left(\Delta \xi_{i} \Delta \xi_{j}\right)\right| & =\max _{1 \leqslant j \leqslant N-1} \sum_{i=1}^{N-1}\left|d_{i, j}\right| \\
& \leqslant\left[\mathrm{O}\left(N^{-1}\right)+\max _{1 \leqslant j \leqslant N-1} \sum_{|i-j|>2} C_{0} N^{-1}(i-j-2)^{-\gamma-2}\right] \\
& =\mathrm{O}\left(N^{-1}\right) .
\end{aligned}
$$

A lower bound is needed for $\operatorname{var}\left(S_{N}\right)=\operatorname{var}\left(\eta_{N}\right)=d_{N}$. Because of (16) and the assumption that $g$ is non-vanishing function

$$
d_{N} \geqslant 2 \sum_{k=1}^{N-1} d_{k, k}^{2} \geqslant \frac{1}{N^{2}} \sum_{k=1}^{N-1} g^{2}\left(\frac{k}{N}\right)+\mathrm{o}\left(\frac{1}{N}\right) \geqslant \frac{1}{N} \int_{0}^{1} g^{2}(t) \mathrm{d} t+\mathrm{o}\left(\frac{1}{N}\right) .
$$

Hence $\operatorname{var} S_{N}=d_{N}>C / N$ for a generic positive constant $C$. The convergence in distribution to a standard Gaussian variable of 


$$
\frac{N^{1-\gamma}\left[\sum_{k=1}^{N-1} \Delta \xi_{k}^{2}-\mathbb{E} \sum_{k=1}^{N-1} \Delta \xi_{k}^{2}\right]}{\sqrt{d}_{N}}
$$

is proved.

Remark 4. If $\gamma>1 / 2$ Theorem 2 is still true even if the process $\xi$ is not centered. It is a consequence of (10) and of $d_{N}>C / N$.

Let us now see when the bias term $\mathbb{E}\left[\eta_{N}\right]-\int_{0}^{1} g(t) \mathrm{d} t$ is negligible. Assumptions (2b') and (32) are introduced below to ensure the convergence of $N d_{N}$. One can check that there are fulfilled for the fractional Brownian motion, fractional Brownian sheet is another instance where the following theorem can be applied.

Theorem 3. Let $\xi$ be a centered Gaussian process such that assumptions (2a) is fulfilled.

Assumption (2b) is replaced by

$\left(2 \mathrm{~b}^{\prime}\right)$ There exist two functions $c_{1}(s), c_{2}(t)$ such that $\partial^{4}\left(r-c_{1}-c_{2}\right) / \partial s^{2} \partial t^{2}$ exists on $[0,1]^{2} \backslash\{(u, u): 0 \leqslant u \leqslant 1\}$. Let $T^{+}=\{(s, t): 0 \leqslant s \leqslant t \leqslant 1\}$ be the upper triangle in $[0,1]^{2}$ (resp. $T^{-}=\{(s, t): 0 \leqslant t \leqslant s \leqslant 1\}$ be the lower triangle) and we assume the existence of a continuous function $C_{+}$on $T^{+}$(respectively $C_{-}$on $T^{-}$) such that

$$
|s-t|^{\gamma+2} \frac{\partial^{4}\left(r-c_{1}-c_{2}\right)}{\partial s^{2} \partial t^{2}}(s, t)=C_{+}(s, t), \quad s, t \in\{(s, t): 0 \leqslant s<t \leqslant 1\}
$$

(resp. $C_{-}$on $\{(s, t): 0 \leqslant t<s \leqslant 1\}$ ).

The assumption (2c) is replaced by:

let us suppose that there exists a function $g$ such that

$$
\sup _{t \in[h, 1-h]}\left|\frac{\delta_{1}^{h} \circ \delta_{2}^{h} r(t, t)}{|h|^{2-\gamma}}-g(t)\right|<C h^{\epsilon+1 / 2}
$$

where $\epsilon>0, h \rightarrow 0, C$ is a generic constant. Let us assume that $g$ is a $1 / 2+\epsilon$ Hölder continuous nonvanishing function.

Moreover we assume that there exists a function $\tilde{g}$

$$
\lim _{h \rightarrow 0^{+}} \sup _{t \in[h, 1-2 h]}\left|\frac{\delta_{1}^{h} \circ \delta_{2}^{h} r(t+h, t)}{|h|^{2-\gamma}}-\tilde{g}(t)\right|=0 .
$$

Then

$$
\sqrt{N}\left(N^{1-\gamma} \sum_{k=1}^{N-1} \Delta \xi_{k}^{2}-\int_{0}^{1} g(t) \mathrm{d} t\right)
$$

converges to a centered Gaussian random variable.

Proof. The bound (31) and (15) yield for $h=\frac{1}{N}$ :

$$
\left|\mathbb{E}\left[\eta_{N}\right]-\frac{1}{N} \sum_{k=1}^{N-1} g\left(\frac{k}{N}\right)\right|<\frac{C}{N^{\epsilon+1 / 2}} .
$$


Since $g$ is Hölder continuous

$$
\left|\frac{1}{N} \sum_{k=1}^{N-1} g\left(\frac{k}{N}\right)-\int_{0}^{1} g(t) \mathrm{d} t\right|<\frac{C}{N^{\epsilon+1 / 2}} .
$$

Let us show that $\lim _{N \rightarrow \infty} N d_{N}$ exists. Recall (12)

$$
d_{N}=2 \sum_{k=1}^{N-1} d_{k, k}^{2}+4 \sum_{1 \leqslant k<j \leqslant N-1} d_{j, k}^{2} .
$$

Because of (16)

$$
\lim _{N \rightarrow \infty} N \sum_{k=1}^{N-1} d_{k, k}^{2}=\int_{0}^{1} g^{2}(t) \mathrm{d} t
$$

Let us now consider the case when $j-k \geqslant 3$. In this case because of (14) and (22)

$$
N d_{j, k}=N^{2-\gamma} \int_{j / N}^{j+1 / N} \mathrm{~d} u \int_{u-1 / N}^{u} \mathrm{~d} x \int_{k / N}^{k+1 / N} \mathrm{~d} v \int_{v-1 / N}^{v} \mathrm{~d} y \frac{C_{+}(x, y)}{(x-y)^{\gamma+2}} .
$$

Then

$$
\left|N d_{j, k}-N^{2-\gamma} \int_{j / N}^{j+1 / N} \mathrm{~d} u \int_{u-1 / N}^{u} \mathrm{~d} x \int_{k / N}^{k+1 / N} \mathrm{~d} v \int_{v-1 / N}^{v} \mathrm{~d} y \frac{C_{+}(j / N, k / N)}{(x-y)^{\gamma+2}}\right| \leqslant \frac{\epsilon_{N}}{(j-k-2)^{\gamma+2}}
$$

with $\epsilon_{N} \rightarrow 0$ when $N \rightarrow \infty$. Actually we use $\left|C_{+}(x, y)-C_{+}(j / N, k / N)\right| \rightarrow 0$ uniformly with respect to $j, k$ when $N \rightarrow \infty$. Moreover

$$
N^{2-\gamma} \int_{j / N}^{j+1 / N} \mathrm{~d} u \int_{u-1 / N}^{u} \mathrm{~d} x \int_{k / N}^{k+1 / N} \mathrm{~d} v \int_{v-1 / N}^{v} \mathrm{~d} y \frac{1}{(x-y)^{\gamma+2}}
$$

is a function of $j-k$ that will be denoted by $f(j-k)$ such that

$$
|f(j-k)| \leqslant \frac{M}{(j-k-2)^{\gamma+2}}
$$

for $M$ a generic constant. Then

$$
\begin{aligned}
N \sum_{1 \leqslant k<j-2 \leqslant N-4} d_{j, k}^{2} & =\frac{1}{N} \sum_{1 \leqslant k<j-2 \leqslant N-4} C_{+}^{2}\left(\frac{j}{N}, \frac{k}{N}\right) f^{2}(j-k) \\
& =\sum_{l=3}^{N-3} f^{2}(l)\left(\frac{1}{N} \sum_{k=1}^{N-1-l} C_{+}^{2}\left(l+\frac{k}{N}, \frac{k}{N}\right)\right) .
\end{aligned}
$$

When $l$ is fixed one can show that

$$
\lim _{N \rightarrow \infty} \frac{1}{N} \sum_{k=1}^{N-1-l} C_{+}^{2}\left(l+\frac{k}{N}, \frac{k}{N}\right)=\int_{0}^{1} C_{+}^{2}(x, x) \mathrm{d} x
$$


and since $\sum_{l=3}^{N-3} f^{2}(l)<+\infty$, the limit of $N \sum_{1 \leqslant k<j-2 \leqslant N-4} d_{j, k}^{2}$ exists. At this point we are reduced to prove the convergence $N \sum_{1 \leqslant k \leqslant N-2} d_{k+2, k}^{2}$ and $N \sum_{1 \leqslant k \leqslant N-1} d_{k+1, k}^{2}$. For the first limit one uses a perturbation argument that can be found in [8], and (32) yields the existence of the second limit. Similar arguments hold when $k>j$ and we skip the technical details.

\section{Application to fractional Brownian sheet}

\subsection{Application of Theorem 1}

In this part, theorems of Section 2 are applied to the identification of the axes of the fractional Brownian sheet. They are identified with the help of generalized quadratic variations of the fractional Brownian sheet restricted to some segments. The geometry of the problem is described in Fig. 1.

In this paper radial segments $[A, B]$ with length $L$ are considered. The distance of the segment to the origin $\mathrm{O}$ is $L \varepsilon>0$ (see Section 3.2 when $\varepsilon=0$ ). The angle of $[A, B]$ with respect to the axes of observations $O x y$ is $\beta$ a parameter under our control. The oriented angle $\theta$ between $O x y$ and $O x^{\prime} y^{\prime}$, the true axes of the fractional Brownian sheet, is the parameter we want to estimate. Let us recall the correlation function of the fractional Brownian sheet $\left(W_{H_{1}, H_{2}}\left(x^{\prime}, y^{\prime}\right)\right)_{\left(x^{\prime}, y^{\prime}\right) \in \mathbb{R}^{2}}$ :

$$
E\left[W\left(x_{1}^{\prime}, y_{1}^{\prime}\right) \cdot W\left(x_{2}^{\prime}, y_{2}^{\prime}\right)\right]=\frac{1}{4}\left(\left|x_{1}^{\prime}\right|^{2 H_{1}}+\left|x_{2}^{\prime}\right|^{2 H_{1}}-\left|x_{1}^{\prime}-x_{2}^{\prime}\right|^{2 H_{1}}\right)\left(\left|y_{1}^{\prime}\right|^{2 H_{2}}+\left|y_{2}^{\prime}\right|^{2 H_{2}}-\left|y_{1}^{\prime}-y_{2}^{\prime}\right|^{2 H_{2}}\right)
$$

where $0<H_{1}, H_{2}<1$.

Let us discuss why we consider $\theta$ as an oriented angle and what is the range of $\theta$. It is obvious that the distribution of the fractional Brownian sheet is invariant with respect to the transformation $y^{\prime} \mapsto-y^{\prime}$. Hence, one can always assume that the orthonormal frames $O x y$ and $O x^{\prime} y^{\prime}$, have the same orientation. Moreover, since the distribution of the fractional Brownian sheet is invariant with respect to the transformation $x^{\prime} \mapsto-x^{\prime}$ we are actually

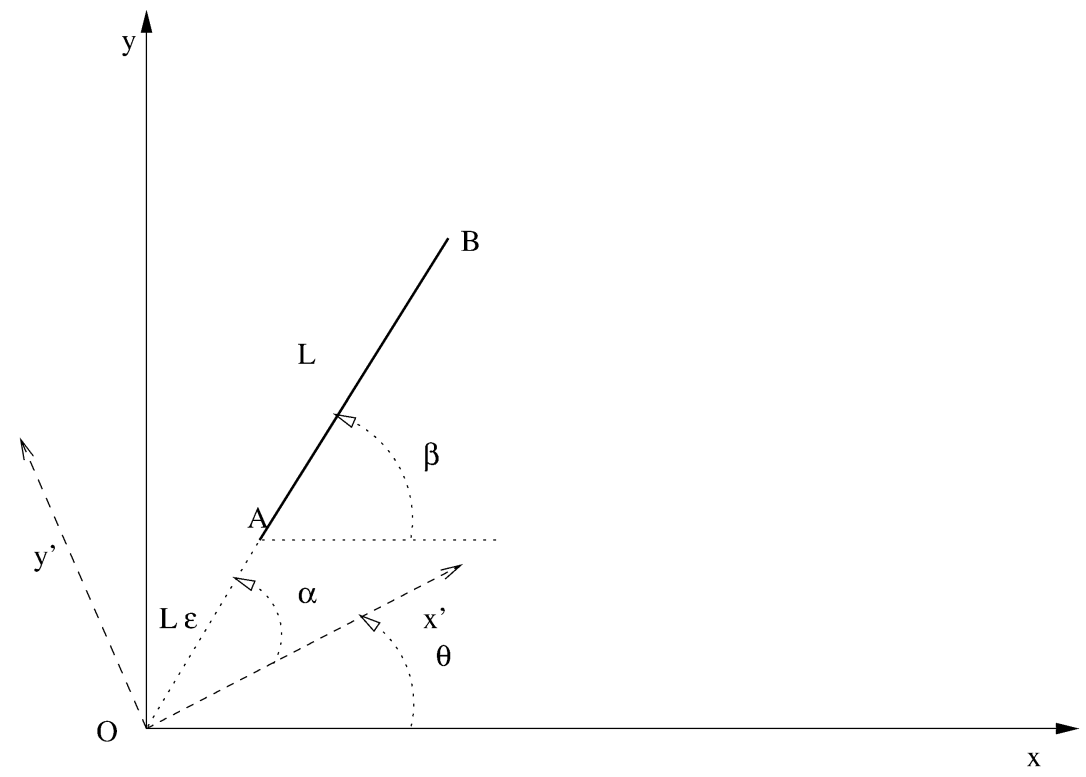

Fig. 1. Geometry of the observations. 
interested in the angle between the two lines $O x$ and $O x^{\prime}$ with no privileged sense $x^{\prime}>0$. Since we are working with oriented frames, it means that one can always assume that $\theta \in\left(-\frac{\pi}{2}, \frac{\pi}{2}\right]$.

Let us denote the oriented angles $\beta$ and $\theta$ as shown in Fig. 1. Then, $\alpha=\theta-\beta$ is the angle of $[A, B]$ with respect to the true axes of $O x^{\prime} y^{\prime}$ of the fractional Brownian sheet.

Let us call $\left(Z_{t}, t \in[0,1]\right)$ the restriction of the fractional Brownian sheet to segment $[A, B]$, which can be parameterized as follows:

$$
\begin{aligned}
x_{t}^{\prime} & =L(t+\varepsilon) \cos \alpha, \\
y_{t}^{\prime} & =L(t+\varepsilon) \sin \alpha,
\end{aligned}
$$

$Z_{t}=W\left(x_{t}^{\prime}, y_{t}^{\prime}\right)$ for $t \in[0,1]$. Then the covariance of $Z, r(s, t)=E\left(Z_{s} Z_{t}\right)$, is

$$
\begin{aligned}
r(s, t)= & \frac{1}{4} L^{2\left(H_{1}+H_{2}\right)}|\cos \alpha|^{2 H_{1}}|\sin \alpha|^{2 H_{2}}\left(|t+\varepsilon|^{2 H_{1}}+|s+\varepsilon|^{2 H_{1}}-|t-s|^{2 H_{1}}\right) \\
& \times\left(|t+\varepsilon|^{2 H_{2}}+|s+\varepsilon|^{2 H_{2}}-|t-s|^{2 H_{2}}\right) .
\end{aligned}
$$

By applying Theorem 1 we will prove the following result.

\section{Proposition 1.}

$$
\lim _{N \rightarrow \infty} N^{2 H_{1}-1} \sum_{k=1}^{N-1}\left(Z_{(k+1) / N}-2 Z_{k / N}+Z_{(k-1) / N}\right)^{2}=\int_{0}^{1} g(t) \mathrm{d} t
$$

almost surely where:

$$
g(t)=\left(4-2^{2 H_{1}}\right) L^{2\left(H_{1}+H_{2}\right)}|\cos \alpha|^{2 H_{1}}|\sin \alpha|^{2 H_{2}}(t+\varepsilon)^{2 H_{2}}
$$

when $H_{1}<H_{2}$ and

$$
g(t)=2\left(4-2^{2 H_{1}}\right) L^{4 H_{1}}|\cos \alpha|^{2 H_{1}}|\sin \alpha|^{2 H_{1}}(t+\varepsilon)^{2 H_{1}}
$$

when $H_{1}=H_{2}$.

Proof. We have to check assumptions of Theorem 1. First $Z$ is a centered Gaussian process and clearly the covariance function is continuous on $[0,1]^{2}$.

Concerning assumption (2b), the partial derivatives of $r$ exist in $[0,1]^{2} \backslash\{(u, u): 0 \leqslant u \leqslant 1\}$, let us now check (3) for $\gamma=2\left(1-H_{1}\right)$ and $c_{1}=c_{2}=0$. Since $r$ is up to a multiplicative constant the product of $f_{i}(t, s)=\left(|t+\varepsilon|^{2 H_{i}}+\right.$ $\left.|s+\varepsilon|^{2 H_{i}}-|t-s|^{2 H_{i}}\right)$, for $i=1,2$, by Leibnitz rule it is enough to have bounds on partial derivatives of the $f_{i}$ 's. Elementary computations postponed to the Appendix yields the bound for $C_{0}$ :

$$
\begin{aligned}
C_{0}(\varepsilon) \leqslant & \frac{1}{4} L^{2\left(H_{1}+H_{2}\right)}|\cos \alpha|^{2 H_{1}}|\sin \alpha|^{2 H_{2}}\left[4(1+\varepsilon)^{2 H_{2}}\right. \\
& +8 \frac{2}{3 \sqrt{3}}\left(1+\max \left(\varepsilon^{2 H_{1}-1},(1+\varepsilon)^{2 H_{1}-1}, \varepsilon^{2 H_{2}-1},(1+\varepsilon)^{2 H_{2}-1}\right)\right) \\
& \left.+8 \frac{2}{3 \sqrt{3}} \max \left(1,(1+\varepsilon)^{2 H_{1}-1},(1+\varepsilon)^{2 H_{2}-1}\right)+8 \max \left(1, \varepsilon^{2 H_{2}-2}, \varepsilon^{2 H_{1}-2}\right)+16\right] .
\end{aligned}
$$

Let us denote

$$
C\left(\alpha, H_{1}, H_{2}, L\right)=\frac{1}{4} L^{2\left(H_{1}+H_{2}\right)}|\cos \alpha|^{2 H_{1}}|\sin \alpha|^{2 H_{2}} .
$$


Thus we have in case $\varepsilon=1$ :

$$
C_{0}(1) \leqslant C\left(\alpha, H_{1}, H_{2}, L\right)\left(40+\frac{80}{3 \sqrt{3}}\right)
$$

where $C_{0}(1)$ is a constant for inequality (3).

Let us now check assumption (2c) for $t+\varepsilon \geqslant h \geqslant 0$

$$
\left(t+\varepsilon+\delta_{j} h\right)^{2 H_{i}}=(t+\varepsilon)^{2 H_{i}}+2 H_{i}(t+\varepsilon)^{2 H_{i}-1} \delta_{j} h+\mathrm{O}\left((t+\varepsilon)^{2 H_{i}-2} h^{2}\right)
$$

for $i=1,2 ; j=1,2,3, \delta_{j}=+1,-1,0$. Moreover

$$
\begin{aligned}
f_{i}\left(t+\delta_{j} h, t+\delta_{k} h\right)= & 2(t+\varepsilon)^{2 H_{i}}+2 H_{i}\left(\delta_{j}+\delta_{k}\right)(t+\varepsilon)^{2 H_{i}-1} h-\left(\left|\delta_{j}-\delta_{k}\right| h\right)^{2 H_{i}} \\
& +H_{i}\left(2 H_{i}-1\right)(t+\varepsilon)^{2 H_{i}-2}\left(\delta_{j}^{2}+\delta_{k}^{2}\right) h^{2}+\mathrm{O}\left(h^{3}\right)(t+\varepsilon)^{2 H_{i}-3} .
\end{aligned}
$$

This yields an asymptotic expansion for $r\left(t+\delta_{j} h, t+\delta_{k} h\right)$ and because of (9) one gets for $h>0$

$$
\begin{aligned}
\delta_{2}^{h} \circ \delta_{1}^{h} r(t, t)= & 4 C\left(\alpha, H_{1}, H_{2}, L\right) h^{2 H_{1}}(t+\varepsilon)^{2 H_{2}}\left(4-2^{2 H_{1}}\right) \\
& \times\left[1+\mathrm{O}\left(h^{2\left(H_{2}-H_{1}\right)}\right)+\mathrm{O}\left(h^{2\left(1-H_{1}\right)}\right)+\mathrm{O}(h)\right]
\end{aligned}
$$

if $H_{1}<H_{2}$. Then, if we set

$$
\begin{aligned}
& g(t)=\left(4-2^{2 H_{1}}\right) L^{2 H}|\cos \alpha|^{2 H_{1}}|\sin \alpha|^{2 H_{2}}(t+\varepsilon)^{2 H_{2}}, \\
& \left|\frac{\delta_{1}^{h} \circ \delta_{2}^{h} r(t, t)}{h^{2-\gamma}}-g(t)\right|=\mathrm{O}\left(h^{2\left(H_{2}-H_{1}\right)}(t+\varepsilon)^{2 H_{1}}\right),
\end{aligned}
$$

which establishes (6).

Whereas when $H_{1}=H_{2}$,

$$
\delta_{2}^{h} \circ \delta_{1}^{h} r(t, t)=8 C\left(\alpha, H_{1}, H_{1}, L\right) h^{2 H_{1}}(t+\varepsilon)^{2 H_{1}}\left(4-2^{2 H_{1}}\right)\left[1+\mathrm{O}\left(h^{2\left(1-H_{1}\right)}\right)+\mathrm{O}(h)\right]
$$

and

$$
g(t)=2\left(4-2^{2 H_{1}}\right) L^{2 H}|\cos \alpha|^{2 H_{1}}|\sin \alpha|^{2 H_{1}}(t+\varepsilon)^{2 H_{1}}
$$

and

$$
\left|\frac{\delta_{1}^{h} \circ \delta_{2}^{h} r(t, t)}{h^{2-\gamma}}-g(t)\right|=\mathrm{O}\left(h^{2-2 H_{1}}(t+\varepsilon)^{4 H_{1}-2}\right) .
$$

Let us denote by

$$
V_{N}(\beta, L, \varepsilon)=\sum_{k=1}^{N-1}\left(Z_{(k+1) / N}-2 Z_{k / N}+Z_{(k-1) / N}\right)^{2}
$$

and state a central limit theorem for $V_{N}(\beta, L, \varepsilon)$.

Proposition 2. If $H_{1}+1 / 4<H_{2}$ then

$$
D\left(H_{1}, H_{2}, \alpha, L, \varepsilon\right)=\left(4-2^{2 H_{1}}\right) L^{2\left(H_{1}+H_{2}\right)}|\cos \alpha|^{2 H_{1}}|\sin \alpha|^{2 H_{2}} \frac{(1+\varepsilon)^{2 H_{2}+1}-\varepsilon^{2 H_{2}+1}}{2 H_{2}+1}
$$

then

$$
\sqrt{N}\left(N^{2 H_{1}-1} V_{N}(\beta, L, \varepsilon)-D\left(H_{1}, H_{2}, \theta-\beta, L, \varepsilon\right)\right)
$$


converges to a standard centered Gaussian random variable.

If $H_{1}=H_{2}, H_{1}<3 / 4$ then

$$
\begin{aligned}
\widetilde{D}\left(H_{1}, \alpha, L, \varepsilon\right)= & 2\left(4-2^{2 H_{1}}\right) L^{4 H_{1}}|\cos \alpha|^{2 H_{1}}|\sin \alpha|^{2 H_{1}} \frac{(1+\varepsilon)^{2 H_{1}+1}-\varepsilon^{2 H_{1}+1}}{2 H_{1}+1} \\
& \times \sqrt{N}\left(N^{2 H_{1}-1} V_{N}(\beta, L, \varepsilon)-\widetilde{D}\left(H_{1}, \theta-\beta, L, \varepsilon\right)\right)
\end{aligned}
$$

converges to a centered Gaussian random variable.

Proof. When $H_{1}+1 / 4<H_{2}$, (31) is satisfied because $2\left(H_{2}-H_{1}\right)>1 / 2$ and we have (41). Moreover $g$ is continuously differentiable. Since $g$ is non-vanishing and since

$$
\int_{0}^{1} g(t) \mathrm{d} t=\left(4-2^{2 H_{1}}\right) L^{2\left(H_{1}+H_{2}\right)}|\cos \alpha|^{2 H_{1}}|\sin \alpha|^{2 H_{2}} \frac{(1+\varepsilon)^{2 H_{2}+1}-\varepsilon^{2 H_{2}+1}}{2 H_{2}+1},
$$

one can check (32) with an asymptotic development of $\delta_{2}^{h} \circ \delta_{1}^{h} r(t+h, t)$ similar to (40). The existence of $C_{+}$and $C_{-}$is a consequence of the computation of $\frac{\partial^{4} r}{\partial s^{2} \partial t^{2}}(s, t)$ done in the appendix. One can apply Theorem 3. The first part of the proposition is proved.

When $H_{1}=H_{2}, 2-2 H_{1}>1 / 2$ and Eq. (43) allow application of Theorem 3 with the same arguments as in the previous case.

Remark 5. Let us define $X_{N}=N^{2 H_{1}-1} V_{N}(\beta, L, \varepsilon)$. Note that with the same arguments, we prove the asymptotic normality of a pair $\left(X_{N}, X_{2 N}\right)$ of rescaled quadratic variations since the central limit theorem can be proved for every linear combination $\lambda X_{N}+\mu X_{2 N}$ which is still a rescaled quadratic variation.

\subsection{Case where $\varepsilon=0$}

In the previous section, the fractional Brownian sheet is restricted to a segment that does not intersect the origin. Technically it yields $\varepsilon>0$. One may wonder what happens when $\varepsilon=0$. Although this choice is in some sense more natural the application of the theorems of the section 2 is more difficult when $\varepsilon=0$. For instance the covariance of $Z$ is no longer differentiable when $t=0$.

In this section one extend the result of Proposition 1 when $\varepsilon=0$. Let us now consider the restriction of the fractional Brownian sheet to $[O, B]$ parameterized by

$$
\begin{gathered}
x_{t}^{\prime}=L t \cos \alpha, \\
y_{t}^{\prime}=L t \sin \alpha, \\
\widetilde{Z}_{t}=W\left(x_{t}^{\prime}, y_{t}^{\prime}\right) \text { for } t \in[0,1],
\end{gathered}
$$

Proposition 3. If $H_{2}>H_{1}>3 / 4$, then

$$
\lim _{N \rightarrow \infty} N^{2 H_{1}-1} \sum_{k=1}^{N-1}\left(\widetilde{Z}_{(k+1) / N}-2 \widetilde{Z}_{k / N}+\widetilde{Z}_{(k-1) / N}\right)^{2}=\frac{\left(4-2^{2 H_{1}}\right) L^{2\left(H_{1}+H_{2}\right)}|\cos \alpha|^{2 H_{1}}|\sin \alpha|^{2 H_{2}}}{2 H_{2}+1} .
$$

If $H_{2}=H_{1}>3 / 4$, then

$$
\lim _{N \rightarrow \infty} N^{2 H_{1}-1} \sum_{k=1}^{N-1}\left(\widetilde{Z}_{(k+1) / N}-2 \widetilde{Z}_{k / N}+\widetilde{Z}_{(k-1) / N}\right)^{2}=\frac{2\left(4-2^{2 H_{1}}\right) L^{4 H_{1}}|\cos \alpha|^{2 H_{1}}|\sin \alpha|^{2 H_{1}}}{2 H_{1}+1} .
$$


Proof. The assumption (2b) is not satisfied for $\widetilde{Z}$. More precisely, the bound obtained in (36) shows that the covariance $r$ of $\widetilde{Z}$ satisfies for $1>\varepsilon>0$ and $s, t \in[\varepsilon, 1]^{2} \backslash\{(u, u): \varepsilon \leqslant u \leqslant 1\}$,

$$
\left|\frac{\partial^{4} r(s, t)}{\partial t^{2} \partial s^{2}}\right| \leqslant \frac{C_{2} \varepsilon^{2 H_{1}-2}}{|s-t|^{\gamma+2}}
$$

for some positive constant $C_{2}$. Let $\varepsilon_{N} \rightarrow+\infty$ be such that $\varepsilon_{N} / N \rightarrow 0$ when $N \rightarrow+\infty$. This yields

$$
\left|d_{j, k}\right| \leqslant \frac{C_{2} N^{-1}}{|j-k-2|^{\gamma+2}}\left(\frac{\varepsilon_{N}}{N}\right)^{2 H_{1}-2} .
$$

One can show as in (24) that

$$
\sum_{\varepsilon_{N} \leqslant k \leqslant j-3 \leqslant N-4} d_{j, k}^{2} \leqslant C_{2}^{2} N^{-2}\left(\frac{\varepsilon_{N}}{N}\right)^{4 H_{1}-4} \sum_{\varepsilon_{N} \leqslant k \leqslant j-3 \leqslant N-4}|j-k-2|^{-2 \gamma-4}=\mathrm{O}\left(\frac{\varepsilon_{N}^{4 H_{1}-4}}{N^{4 H_{1}-3}}\right),
$$

and that

$$
\sum_{1 \leqslant k \leqslant j-3 \leqslant N-4 ; k<\varepsilon_{N}} d_{j, k}^{2} \leqslant C \frac{\varepsilon_{N}}{N},
$$

for a generic constant $C$, because $d_{j, k}=\mathrm{O}(1 / N)$. Let us choose $\varepsilon_{N}=N^{\left(4-4 H_{1}\right) /\left(5-4 H_{1}\right)}$, then $d_{N}=\mathrm{O}\left(N^{1 /\left(4 H_{1}-5\right)}\right)$. Because of (29) we get the almost sure convergence if

$$
\frac{1}{2}<\frac{1}{5-4 H_{1}}
$$

which leads to $H_{1}>3 / 4$. The same arguments hold for $H_{1}=H_{2}>3 / 4$.

\subsection{Estimators of the parameters $H_{1}, H_{2}$ and $\theta$}

Let us recall that $H_{1} \leqslant H_{2}$ where $H_{1}$ is attached to axe $O x^{\prime}$ of the fractional Brownian sheet. In this part, we use previous results to construct consistent estimators of $H_{1}, H_{2}$ and $\theta=\left(O x, O x^{\prime}\right)$, the angle between the $O x^{\prime}$ axis of the fractional Brownian sheet with the observation axis $O x$. Estimators of $H_{1}, H_{2}$ are studied in Propositions 4 and 5, while Proposition 6 gives an estimator of $\theta$.

In the following propositions we assume that the segment is not contained in one of the axes of the fractional Brownian sheet i.e. $\beta \neq \theta+k \pi / 2, \forall k \in \mathbb{Z}$.

Proposition 4. If $\beta \neq \theta+k \pi / 2, \forall k \in \mathbb{Z}$, and if $V_{N}$ is defined by (44)

$$
\widehat{H}_{1 N}=\frac{1+\log \left(V_{N}(\beta, L, \varepsilon) / V_{2 N}(\beta, L, \varepsilon)\right) / \log 2}{2}
$$

and

$$
\widehat{H}_{2 N}=\frac{1}{2 \log 2} \log \left[\frac{V_{N}(\beta, 2 L, \varepsilon)}{V_{N}(\beta, L, \varepsilon)}\right]-\widehat{H}_{1 N}
$$

are respectively strongly consistent estimators of $H_{1}$ and $H_{2}$.

Proof. Because of Proposition 1,

$$
\lim _{N \rightarrow+\infty} \frac{V_{N}(\beta, L, \varepsilon)}{N^{1-2 H_{1}}}=\int_{0}^{1} g(t) \mathrm{d} t .
$$


When $\beta \neq \theta+k \pi / 2, \forall k \in \mathbb{Z}$,

$$
\int_{0}^{1} g(t) \mathrm{d} t \neq 0
$$

and (49) is established.

To prove (50) let us remark that

$$
\frac{V_{N}(\beta, 2 L, \varepsilon)}{V_{N}(\beta, L, \varepsilon)} \rightarrow 2^{2\left(H_{1}+H_{2}\right)}
$$

when $N \rightarrow+\infty$.

Then we can give a confidence interval for the estimation of $H_{1}$.

Proposition 5. Let $H_{1}+1 / 4<H_{2}$, and let $\alpha \neq k \pi / 2, \forall k \in \mathbb{Z}$.

Let $m=D\left(H_{1}, H_{2}, \alpha, L, \varepsilon\right)$ defined in Proposition 2. If $\Gamma$ denotes $\lim _{N \rightarrow \infty} N \mathbb{E}\left(X_{2 N}-X_{N}\right)^{2}$, then $\Gamma>0$ and

$$
\mathbb{P}\left\{2 m \Gamma^{-1 / 2} \log 2 \sqrt{N}\left|H_{1 N}-H_{1}\right| \leqslant 1.96\right\} \rightarrow 0.95
$$

when $N \rightarrow+\infty$.

Proof. Because of Remark $5\left(X_{N}, X_{2 N}\right)$ is asymptotically normal and the $\operatorname{limit}_{N \rightarrow \infty} \lim _{N \rightarrow}\left(X_{2 N}-X_{N}\right)^{2}$ exists. Then $N \mathbb{E}\left(X_{2 N}-X_{N}\right)^{2} \leqslant\left(\frac{3}{2}-\sqrt{2}\right) \lim _{N \rightarrow+\infty} N d_{N}$. Hence, $\Gamma>0$ and because of Slutsky theorem:

$$
\sqrt{N} \log \left(X_{N} / X_{2 N}\right) \rightarrow \mathcal{N}\left(0, \sigma^{2}\right)
$$

when $N \rightarrow \infty$, where the convergence is in distribution, and where

$$
\sigma^{2}=\lim _{N \rightarrow \infty} \frac{N \mathbb{E}\left(X_{2 N}-X_{N}\right)^{2}}{m^{2}} .
$$

Since

$$
\log \left(X_{N} / X_{2 N}\right)=2 \log 2\left(\widehat{H}_{1 N}-H_{1}\right),
$$

and $\sigma^{2}=\Gamma / m^{2}$, the proposition is proved.

Please note that the rate of convergence $\mathrm{O}(1 / \sqrt{N})$ is known to be of the same order as the one given by the Cramér-Rao bound (cf. [6] and [4] when the Hurst exponent of a fractional Brownian Motion is identified). In our case, since the process $Z$ is not with stationary increments, the Cramér-Rao bound seems difficult to estimate.

Let us give a numerical application of the previous proposition. In practice we have to compute $m^{-2} \Gamma$. Actually the computation of $\Gamma$ seems delicate but $N \mathbb{E}\left(X_{2 N}-X_{N}\right)^{2} \leqslant 3 N \mathbb{E}\left(X_{N}-m\right)^{2}=3 N d_{N}$ with $d_{N}$ defined by (12) with $Z$ in place of $\xi$. And we have an upper bound for $N d_{N}$. More precisely, because of (28)

$$
N d_{N} \leqslant 10 M^{2}+\frac{16}{3} C_{0}^{2}(\varepsilon) \text {. }
$$

Moreover $g(1)$ is the supremum of $g$ and we can take

$$
M>4\left(4-2^{2 H_{1}}\right) C\left(\alpha, H_{1}, H_{2}, L\right)(1+\varepsilon)^{2 H_{2}}
$$

(for instance $\left.M=4\left(4-2^{2 H_{1}}\right) C\left(\alpha, H_{1}, H_{2}, L\right)(1+\varepsilon)^{2}\right)$. Let us assume $\varepsilon=1$ and recall that

$$
C_{0}(1) \leqslant\left(40+\frac{80}{3 \sqrt{3}}\right) C\left(\alpha, H_{1}, H_{2}, L\right)
$$


then

$$
N d_{N} \leqslant C^{2}\left(\alpha, H_{1}, H_{2}, L\right)\left[160\left(4-2^{2 H_{1}}\right)^{2} 4+\frac{16}{3}\left(40+\frac{80}{3 \sqrt{3}}\right)^{2}\right] .
$$

Moreover

$$
m=\frac{4\left(4-2^{2 H_{1}}\right)\left(2^{2 H_{2}+1}-1\right) C\left(\alpha, H_{1}, H_{2}, L\right)}{2 H_{2}+1}
$$

for $\varepsilon=1$ and

$$
\begin{aligned}
\frac{\Gamma}{m^{2}} & \leqslant \frac{3\left[160\left(4-2^{2 H_{1}}\right)^{2} 4+(16 / 3)(40+80 /(3 \sqrt{3}))^{2}\right]\left(2 H_{2}+1\right)^{2}}{16\left(4-2^{2 H_{1}}\right)^{2}\left(2^{2 H_{2}+1}-1\right)^{2}} \\
& =3\left[40+\frac{16}{3\left(4-2^{2 H_{1}}\right)^{2}}\left(10+\frac{20}{3 \sqrt{3}}\right)^{2}\right]\left(\frac{2 H_{2}+1}{2^{2 H_{2}+1}-1}\right)^{2} .
\end{aligned}
$$

Let us assume $H_{1}<\frac{1}{2}<H_{2}$ as an example to have an order of how big shall be $N$ to have a meaningful interval. Then, $\left(2 H_{2}+1\right) /\left(2^{2 H_{2}+1}-1\right) \leqslant 1$ and $4-2^{2 H_{1}}>2$.

$$
\frac{1.96 \sqrt{\Gamma}}{m}<58.39
$$

and the confidence interval length is bounded with $58.39 / \sqrt{N}$.

In practical situations, we may assume $H_{1} \neq H_{2}$. In this case we can detect the anisotropy of the fractional Brownian sheet, which means than we can estimate $\theta$.

Proposition 6. If $H_{1} \neq H_{2}, \theta \in(-\pi / 2,0) \cup(0, \pi / 2)$, and $\beta \neq \theta+k \pi / 2, \forall k \in \mathbb{Z}$, set

$$
f_{N}(\beta)=\arctan \left(\left(\frac{V_{N}(\beta+\pi / 2, L, \varepsilon)}{V_{N}(\beta, L, \varepsilon)}\right)^{1 /\left(2\left(\widehat{H_{1 N}}-\widehat{H_{2 N}}\right)\right)}\right)
$$

then

$$
\hat{\theta}_{N}=\operatorname{sign}\left(f_{N}\left(\frac{-\pi}{4}\right)-f_{N}\left(\frac{\pi}{4}\right)\right) f_{N}(0)
$$

is a strongly consistent estimator of $\theta$, where $\operatorname{sign}(x)=1$ if $x \geqslant 0$ and $\operatorname{sign}(x)=-1$ if $x<0$.

Proof. Because of Proposition 1

$$
\frac{V_{N}(\beta+\pi / 2, L, \varepsilon)}{V_{N}(\beta, L, \varepsilon)} \rightarrow|\tan (\theta-\beta)|^{2\left(H_{1}-H_{2}\right)}
$$

when $N \rightarrow+\infty$. Hence, with our assumptions,

$$
f_{N}(\beta) \rightarrow \arctan (|\tan (\theta-\beta)|),
$$

when $N \rightarrow+\infty$. Since $\theta \in(-\pi / 2,0) \cup(0, \pi / 2), f_{N}(0) \rightarrow|\theta|$ when $N \rightarrow+\infty$. Please note that the last assumption is not a real loss of generality since in general we may choose $\theta \in[-\pi / 2, \pi / 2]$. Elementary computations show that

$$
\arctan \left(\left|\tan \left(\theta+\frac{\pi}{4}\right)\right|\right)-\arctan \left(\left|\tan \left(\theta-\frac{\pi}{4}\right)\right|\right)>0
$$

if and only if $\theta \in(0, \pi / 2)$. Since

$$
f_{N}\left(\frac{-\pi}{4}\right)-f_{N}\left(\frac{\pi}{4}\right) \rightarrow \arctan \left(\left|\tan \left(\theta+\frac{\pi}{4}\right)\right|\right)-\arctan \left(\left|\tan \left(\theta-\frac{\pi}{4}\right)\right|\right)
$$


when $N \rightarrow+\infty$, the proof is complete.

Please remark that we do not use all the information available to construct our estimators. This might be an advantage if the parameters are actually functions of the position, which means that the fractional Brownian sheet is actually a first order simplification. To better use the available information we could consider $K$ generalized quadratic variations for different $\beta_{j}, j=1, \ldots, K$, we get estimators $\widehat{H}_{1 N}^{j}, \widehat{H}_{2 N}^{j}, \hat{\theta}_{n}^{j}$. Thus, we could propose a more robust version of the estimators as the empirical means of these estimators. Other problems may also be addressed in the future such as estimation of the variance of the estimators proposed above.

\section{Appendix}

Let us prove the upper bound (36). For $1 \geqslant s>t \geqslant 0$,

$$
\begin{aligned}
& \frac{\partial f_{i}}{\partial t}(t, s)=2 H_{i}\left((t+\varepsilon)^{2 H_{i}-1}+(s-t)^{2 H_{i}-1}\right), \\
& \frac{\partial f_{i}}{\partial s}(t, s)=2 H_{i}\left((s+\varepsilon)^{2 H_{i}-1}-(s-t)^{2 H_{i}-1}\right), \\
& \frac{\partial^{2} f_{i}}{\partial t^{2}}(t, s)=2 H_{i}\left(2 H_{i}-1\right)\left[(t+\varepsilon)^{2 H_{i}-2}-(s-t)^{2 H_{i}-2}\right], \\
& \frac{\partial^{2} f_{i}}{\partial s^{2}}(t, s)=2 H_{i}\left(2 H_{i}-1\right)\left[(s+\varepsilon)^{2 H_{i}-2}-(s-t)^{2 H_{i}-2}\right], \\
& \frac{\partial^{2} f_{i}}{\partial s \partial t}(t, s)=2 H_{i}\left(2 H_{i}-1\right)(s-t)^{2 H_{i}-2}, \\
& \frac{\partial^{3} f_{i}}{\partial s^{2} \partial t}(t, s)=2 H_{i}\left(2 H_{i}-1\right)\left(2 H_{i}-2\right)(s-t)^{2 H_{i}-3}, \\
& \frac{\partial^{4} f_{i}}{\partial s^{2} \partial t^{2}}(t, s)=-2 H_{i}\left(2 H_{i}-1\right)\left(2 H_{i}-2\right)\left(2 H_{i}-3\right)(s-t)^{2 H_{i}-4}
\end{aligned}
$$

for $i=1,2$. Hence:

$$
\begin{aligned}
(s-t)^{\gamma+2} \frac{\partial^{4} f_{1}}{\partial s^{2} \partial t^{2}} f_{2}= & -2 H_{1}\left(2 H_{1}-1\right)\left(2 H_{1}-2\right)\left(2 H_{1}-3\right) f_{2}, \\
(s-t)^{\gamma+2} \frac{\partial^{3} f_{1}}{\partial s^{2} \partial t} \frac{\partial f_{2}}{\partial t}= & 2 H_{1}\left(2 H_{1}-1\right)\left(2 H_{1}-2\right)(s-t) 2 H_{2}\left[\left((t+\varepsilon)^{2 H_{2}-1}+(s-t)^{2 H_{2}-1}\right)\right], \\
(s-t)^{\gamma+2} \frac{\partial^{3} f_{1}}{\partial t^{2} \partial s} \frac{\partial f_{2}}{\partial t}= & -2 H_{1}\left(2 H_{1}-1\right)\left(2 H_{1}-2\right)(s-t) 2 H_{2}\left[\left((s+\varepsilon)^{2 H_{2}-1}-(s-t)^{2 H_{2}-1}\right)\right], \\
(s-t)^{\gamma+2} \frac{\partial^{2} f_{1}}{\partial s^{2}} \frac{\partial^{2} f_{2}}{\partial t^{2}}= & 4 \prod_{i=1,2} H_{i}\left(2 H_{i}-1\right)\left[(s+\varepsilon)^{2 H_{1}-2}(s-t)^{2-H_{1}}-(s-t)^{H_{1}}\right] \\
& \times\left[(t+\varepsilon)^{2 H_{2}-2}(s-t)^{2-H_{1}}-(s-t)^{2 H_{2}-H_{1}}\right], \\
(s-t)^{\gamma+2} \frac{\partial^{2} f_{1}}{\partial s \partial t} \frac{\partial^{2} f_{2}}{\partial s \partial t}= & 4 \prod_{i=1,2} H_{i}\left(2 H_{i}-1\right)(s-t)^{2 H_{2}}
\end{aligned}
$$

are bounded on $[0,1]^{2}$, and consequently assumption (2b) is fulfilled. More precisely, since $\left.H_{i} \in\right] 0,14[$, for $i=1,2$ : 
$\left|2 H_{i}\right| \leqslant 2$,

$\left|2 H_{i}\left(2 H_{i}-1\right)\right| \leqslant 2$,

$\left|2 H_{i}\left(2 H_{i}-1\right)\left(2 H_{i}-2\right)\right| \leqslant \frac{2}{3 \sqrt{3}}$,

$\left|2 H_{i}\left(2 H_{i}-1\right)\left(2 H_{i}-2\right)\left(2 H_{i}-3\right)\right| \leqslant 1$

and

$$
\begin{aligned}
& \sup _{0 \leqslant t<s \leqslant 1}\left|(t+\varepsilon)^{2 H_{i}}+(s+\varepsilon)^{2 H_{i}}-(s-t)^{2 H_{i}}\right|=2(1+\varepsilon)^{2 H_{i}}, \\
& \sup _{0 \leqslant t<s \leqslant 1}\left|\left((s-t)(t+\varepsilon)^{2 H_{i}-1}+(s-t)^{2 H_{i}}\right)\right| \leqslant\left(1+\max \left(\varepsilon^{2 H_{i}-1},(1+\varepsilon)^{2 H_{i}-1}\right)\right), \\
& \sup _{0 \leqslant t<s \leqslant 1}\left|\left((s-t)(t+\varepsilon)^{2 H_{i}-1}-(s-t)^{2 H_{i}}\right)\right| \leqslant \max \left(1,(1+\varepsilon)^{2 H_{i}-1}\right), \\
& \sup _{0 \leqslant t<s \leqslant 1}\left|(s+\varepsilon)^{2 H_{i}-2}(s-t)^{2-H_{i}}-(s-t)^{H_{i}}\right|=\sup _{0 \leqslant t<s \leqslant 1}\left|(s-t)^{H_{i}}\left[1-\left(\frac{s-t}{s+\varepsilon}\right)^{2-2 H_{i}}\right]\right| \leqslant 1, \\
& \sup _{0 \leqslant s<t \leqslant 1}\left|(s+\varepsilon)^{2 H_{1}-2}(s-t)^{2-H_{1}}-(s-t)^{H_{1}}\right| \leqslant \max \left(1, \varepsilon^{2 H_{1}-2}\right), \\
& \sup _{0 \leqslant t<s \leqslant 1}\left|(t+\varepsilon)^{2 H_{2}-2}(s-t)^{2-H_{1}}-(s-t)^{2 H_{2}-H_{1}}\right|=\sup _{0 \leqslant t<s \leqslant 1}\left|(s-t)^{2 H_{2}-H_{1}}\left[1-\left(\frac{s-t}{t+\varepsilon}\right)^{2-2 H_{2}}\right]\right| \\
& \quad \leqslant \max \left(1, \varepsilon^{2 H_{2}-2}\right), \\
& \sup _{0 \leqslant t<s \leqslant 1}(s-t)^{2 H_{i}}=1 .
\end{aligned}
$$

The fourth derivative of $r$ is the sum of terms of the following form:

$$
\begin{aligned}
& (s-t)^{\gamma+2}\left(\frac{\partial^{4}}{\partial s^{2} \partial t^{2}} f_{1} \cdot f_{2}+\frac{\partial^{4}}{\partial s^{2} \partial t^{2}} f_{2} \cdot f_{1}\right) \leqslant 4(1+\varepsilon)^{2 H_{2}}, \\
& (s-t)^{\gamma+2} \frac{\partial^{3}}{\partial s^{2} \partial t} f_{1} \cdot \frac{\partial}{\partial t} f_{2} \leqslant \frac{4}{3 \sqrt{3}}\left(1+\max \left(\varepsilon^{2 H_{1}-1},(1+\varepsilon)^{2 H_{1}-1}\right)\right)
\end{aligned}
$$

two of them are in this form, similarly

$$
\begin{aligned}
& (s-t)^{\gamma+2} \frac{\partial^{3}}{\partial s^{2} \partial t} f_{2} \cdot \frac{\partial}{\partial t} f_{1} \leqslant \frac{4}{3 \sqrt{3}}\left(1+\max \left(\varepsilon^{2 H_{2}-1},(1+\varepsilon)^{2 H_{2}-1}\right)\right), \\
& (s-t)^{\gamma+2} \frac{\partial^{3}}{\partial t^{2} \partial s} f_{1} \cdot \frac{\partial}{\partial s} f_{2} \leqslant \frac{4}{3 \sqrt{3}} \max \left(1,(1+\varepsilon)^{2 H_{2}-1}\right)
\end{aligned}
$$

two of them are in this form, similar terms exist when we interchange $f_{1}$ and $f_{2}$,

$$
(s-t)^{\gamma+2} \frac{\partial^{2}}{\partial s^{2}} f_{1} \cdot \frac{\partial^{2}}{\partial t^{2}} f_{2} \leqslant 4 \max \left(1, \varepsilon^{2 H_{2}-2}, \varepsilon^{2 H_{1}-2}\right)
$$

and two of them are in this form, so they add $8 \max \left(1, \varepsilon^{2 H_{2}-2}, \varepsilon^{2 H_{1}-2}\right)$,

$$
(s-t)^{\gamma+2} \frac{\partial^{2}}{\partial s \partial t} f_{1} \cdot \frac{\partial^{2}}{\partial s \partial t} f_{2} \leqslant 4
$$

and four of them are in this form, so they add 16. 
This sum of terms has to be multiplied by the coefficient

$$
\frac{1}{4} L^{2\left(H_{1}+H_{2}\right)}|\cos \alpha|^{2 H_{1}}|\sin \alpha|^{2 H_{2}}
$$

and

$$
\begin{aligned}
C_{0}(\varepsilon) \leqslant & \frac{1}{4} L^{2\left(H_{1}+H_{2}\right)}|\cos \alpha|^{2 H_{1}}|\sin \alpha|^{2 H_{2}}\left[4(1+\varepsilon)^{2 H_{2}}\right. \\
& +8 \frac{2}{3 \sqrt{3}}\left(1+\max \left(\varepsilon^{2 H_{1}-1},(1+\varepsilon)^{2 H_{1}-1}, \varepsilon^{2 H_{2}-1},(1+\varepsilon)^{2 H_{2}-1}\right)\right) \\
& \left.+8 \frac{2}{3 \sqrt{3}} \max \left(1,(1+\varepsilon)^{2 H_{1}-1},(1+\varepsilon)^{2 H_{2}-1}\right)+8 \max \left(1, \varepsilon^{2 H_{2}-2}, \varepsilon^{2 H_{1}-2}\right)+16\right] .
\end{aligned}
$$

\section{References}

[1] A. Ayache, S. Léger, M. Pontier, Drap brownien fractionnaire, Potential Anal. 17 (1) (2002) 31-43.

[2] G. Baxter, A strong limit theorem for Gaussian processes, Proc. Amer. Soc. 7 (1956) 522-527.

[3] A. Benassi, S. Cohen, J. Istas, S. Jaffard, Identification of filtered white noises, Stochastic Process. Appl. 75 (1998) 31-49.

[4] J.-F. Coeurjolly, J. Istas, Cramér-Rao bounds for fractional Brownian motions, Statist. Probab. Lett. (2001).

[5] S. Cohen, X. Guyon, O. Perrin, M. Pontier, Identification of an isometric transformation of the standard Brownian sheet, à paraître au J. Statist. Plann. Inference, 2004.

[6] R. Dahlhaus, Efficient parameter estimation for self-similar processes, Ann. Statist. 17 (1989) 1749-1766.

[7] D. Feyel, A. de La Pradelle, On fractional Brownian processes, Potential Anal. 10 (3) (1999) 273-288.

[8] E.G. Gladyshev, A new limit theorem for Gaussian process, Theory Probab. Appl. 6 (1961) 52-61.

[9] X. Guyon, J. Léon, Convergence en loi des H-variations d'un processus gaussien stationnaire, Ann. Inst. H. Poincaré 25 (1989) $265-282$.

[10] J. Istas, G. Lang, Quadratic variations and estimation of the Hölder index of a Gaussian process, Ann. Inst. H. Poincaré 33 (4) (1997) $407-436$.

[11] A. Kamont, On the fractional anisotropic Wiener field, Probab. Math. Statist. 18 (1996) 85-98.

[12] R. Klein, E. Giné, On quadratic variations of processes with Gaussian increments, Ann. Probab. 3 (4) (1975) $716-721$.

[13] S. Léger, Analyse stochastique de signaux multi-fractaux et estimations de paramètres, PhD thesis, Université d'Orléans, 2000.

[14] O. Perrin, Quadratic variation for Gaussian processes and application to time deformation, Stochastic Process. Appl. 82 (2) (1999) 293305. 